\title{
Numerical transient hygro-elastic analyses of reinforced Fickian and non-Fickian polymers
}

\author{
T. Peret ${ }^{\mathrm{a}, \mathrm{b}}$, A. Clement ${ }^{\mathrm{b}, *}$, S. Freour ${ }^{\mathrm{b}}, \mathrm{F}$. Jacquemin ${ }^{\mathrm{b}}$ \\ ${ }^{a}$ Institut de Recherche Technologique Jules Verne, Chemin du Chaffault, 44340 Bouguenais, France \\ ${ }^{\mathrm{b}}$ Institut de Recherche en Génie Civil et Mécanique, UMR CNRS 6183, Université de Nantes, Centrale Nantes, 58 Rue Michel Ange, BP 420,44606 Saint-Nazaire, France
}

The present paper is devoted to the impact of water diffusion within reinforced polymer composites. The resulting ageing may damage a structure made with these materials which can no longer perform its function. In order to predict the long-term behavior of such materials in humid environments, numerical investigations can be done with the classical Finite Element Method. In this work, the FEM is used to solve uncoupled mechanical-water diffusion boundary problems. Simulations are done at the microscopic level in order to take into account the heterogeneity of the composite materials. Both the matrix and the reinforcements are assumed linear elastic materials. Two historical models of water diffusion are studied: Fick and Langmuir.
Keywords:

Composite materials

Water diffusion models

Finite Element Method

Internal stresses

Transient hygro-mechanical problem

\section{Introduction}

Nowadays, the use of Glass or Carbon Fiber Reinforced Polymer (GFRP/CFRP) is growing in many industrial sectors such as transport and energy. This is especially true in the marine environment with the enthusiasm for the renewable marine energies [1-3]. This interest for composite materials is mainly due to better specific properties than the conventional metallic materials. Indeed, polymer matrix composite materials allow a substantial mass saving leading to a lower fuel consumption [4] or better performances for the energy converting systems [5]. Moreover, composites are fatigue resistant [6,7], they do not suffer corrosion and are easier to repair [4]. In addition, the structure can be monitored in real time using Bragg grating sensors incorporated in the core of the material during manufacture [8,9]. The number of interventions decreases causing an operating cost reduction [4].

Although GFRP and CFRP present many advantages over metallic materials for marine applications, one must be cautious. Indeed, for such materials, it is well known that the polymeric resins (polyester, epoxy, etc.), used as matrix, absorb moisture. This absorption leads to a premature aging of the material. In fact, the absorbed water can react with the polymer network [10] and can decrease the mechanical properties (plasticization, hydrolysis). Furthermore, moisture absorption yields a hygroscopic swelling of the matrix, which, coupled to the contrast between the mechanical

\footnotetext{
* Corresponding author. Tel.: +33 272648742.

E-mail address: alexandre.clement@univ-nantes.fr (A. Clement).
}

properties of the matrix and the reinforcements (assumed hydrophobic), induces stresses within the composite [11]. Those stresses might deteriorate the matrix/reinforcement interface $[12,13]$. Moreover, the latter internal stresses added to the ones induced by external forces lead to reduce the sustainability of the material [14-16], and thus disrupt the behavior of the structure [17,18]. It is thereby essential to perform numerical analysis to predict the long-term behavior of the structure.

A lot of recent experimental studies have been devoted to the diffusion of water in polymeric resins and composites [19-23]. Most of them point out behavior following the Fick model predictions [24] but some exhibit deviations from this reference model, that have as a consequence been called "anomalies of diffusion" $[24,25]$. The modeling of these so-called anomalies of diffusion can be done using improved models such as the Langmuir one [26]. These models can be used to study the water diffusion behavior of a composite structure. The involved boundary value problems can be numerically solved with the classical Finite Element Method (FEM). In [27-29], the authors have carried out such studies assuming the Fick law to be satisfied. However, to the knowledge of the authors, performing a full numerical study with the Langmuir model has not been proposed while it allows representing a more complex physics. Indeed, due to analogies with the thermal linear Fourier law, one can use industrial finite element software to perform analyses with the Fick model. In this work, the Langmuir model was implemented in the Abaqus ${ }^{\mathrm{TM}}$ code under user-element form [30]. Simulations at microscopic scale, where the geometrical details of the heterogeneities appear, were 
performed. For the considered materials, the distribution of the reinforcements is usually not regular $[27,28,31]$ and a random generator is thus used to create geometries of the microstructure when one wants to consider a high volume fraction of fibers. Two diffusion models are considered: the classical Ficks law and the Langmuirs model which assumes that absorbed moisture consists of mobile and bound phases [26]. In this paper, a numerical transient uncoupled hygro-elastic analysis is carried out so as to quantify the diffusion phenomenon. The resulting time-dependent hygroscopic strain field is then used to evaluate the deformation of the material considering a linear elastic assumption.

The paper is organized as follows. Section 2 deals with the diffusion and mechanical constitutive equations. The differences between the two diffusion models are presented. Section 3 gives details concerning the discretized problem within the FEM framework and its resolution. In the same section, the numerical technique involving an unregular fibers distribution in the microstructure is exposed. Sections 4 and 5 are dedicated to the numerical investigations. Two distinct studies are carried out. The first application involves an epoxy based system characterized by a strongly non-Fickian diffusion behavior. The resin serves as a matrix in an unidirectional composite reinforced by long carbon fibers. The impact of the random geometries is discussed through comparisons based on local and global quantities of interest. The second application deals with an epoxy system reinforced with long glass fibers. Unlike the first example the diffusion within the matrix can be well represented with both models. The impact of each model on diffusion and mechanical quantities is then analyzed. Some concluding remarks are finally drawn in Section 6.

\section{Problem formulation}

\subsection{Diffusion problem}

We consider a heterogeneous material, schematically depicted on Fig. 1, which occupies a spatial domain $\Omega=\Omega_{m} \cup \Omega_{r} \in \mathbb{R}^{d}$ with $d \in\{1,2,3\} . \Omega_{m}$ and $\Omega_{r}$ respectively represent the matrix and the reinforcements. The variable $c(\boldsymbol{x}, t)$ denotes the moisture content of a material point, characterized by its position through vector $\boldsymbol{x}$, at time $t$

This moisture content $c(\boldsymbol{x}, t)$ is defined by

$c(\boldsymbol{x}, t)=\frac{m_{w}(\boldsymbol{x}, t)}{m_{0}(\boldsymbol{x})}$,

where $m_{w}(\boldsymbol{x}, t)$ is the local increase in mass of water whereas $m_{0}(\boldsymbol{x})$ is the local mass at the initial time. The spatial average moisture content $\bar{c}(t)$ can be obtained with the following relation

$\bar{c}(t)=\frac{1}{M_{0}} \int_{\Omega} \rho(\boldsymbol{x}) c(\boldsymbol{x}, t) d \Omega$,

where $\rho(\boldsymbol{x})$ is the local density and $M_{0}$ is the mass of the sample at initial time. In the following, $\bar{c}(t)$ will also be referred as the macroscopic or global moisture content. From an experimental point of view, using the knowledge on $M_{0}$ and the mass $M(t)$ at time $t$ of

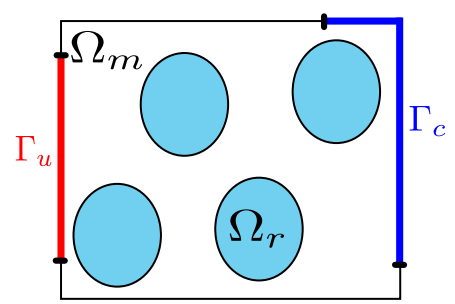

Fig. 1. Model problem. the sample, the overall volume of which is $\Omega, \bar{c}(t)$ is evaluated using the following relationship

$\bar{c}(t)=\frac{M(t)-M_{0}}{M_{0}}=\frac{M_{w}(t)}{M_{0}}$,

where $M_{w}(t)$ represents the global increase in mass of water within the sample with respect to $M_{0}$. From now on, all equations will be written according to the local moisture content $c(\boldsymbol{x}, t)$. In this work, we assume that the diffusion process is governed by a unique diffusion coefficient $D$ in each spatial direction. Moreover, since the fibers are considered hydrophobic, the problem may be solely formulated on the domain $\Omega_{m}$. This remark is true for both models which are exposed in the following.

\subsubsection{Fick diffusion model [24]}

The Fick law is a common model to represent a diffusion process where each water molecule is free to move in the polymer network associated with domain $\Omega_{m}$. Since the diffusivity is assumed constant (i.e. independent of moisture content or mechanical states), the Fick local diffusion problem writes: find the solution field $c(\boldsymbol{x}, t)$ such that it verifies

$$
\begin{aligned}
& \frac{\partial c}{\partial t}=D \Delta c \quad \text { on } \Omega_{m}, \\
& c=c_{\text {imp }} \quad \text { on } \Gamma_{c},
\end{aligned}
$$

where $c_{\text {imp }}$ is a given moisture content applied on a part $\Gamma_{c}$ of boundary $\partial \Omega$.

\subsubsection{Langmuir diffusion model}

In [26], the authors divided the water molecules in two populations. The first $n(\boldsymbol{x}, t)$ is free to move while the molecules of the second phase $N(\boldsymbol{x}, t)$ are bonded to the polymer network due to reversible chemical reactions such as those occurring when moisture induced plasticization takes place. A free water molecule could become a bonded one with a frequency $\alpha$ and reciprocally a bonded molecule could be freed with a frequency $\beta$. The total moisture content $c(\boldsymbol{x}, t)$ naturally verifies

$c(\boldsymbol{x}, t)=n(\boldsymbol{x}, t)+N(\boldsymbol{x}, t)$.

The Langmuir local diffusion problem writes: find the solution fields $n(\boldsymbol{x}, t)$ and $N(\boldsymbol{x}, t)$ such that they verify

$$
\begin{aligned}
& \frac{\partial n}{\partial t}+\frac{\partial N}{\partial t}=D \Delta n \text { on } \Omega_{m}, \\
& \frac{\partial N}{\partial t}=\alpha n-\beta N \text { on } \Omega_{m}, \\
& n=n_{i m p} \text { on } \Gamma_{c}, \\
& N=N_{i m p} \text { on } \Gamma_{c},
\end{aligned}
$$

where $n_{i m p}$ and $N_{\text {imp }}$ are respectively the imposed free and bounded moisture content on $\Gamma_{c}$. Considering those two different populations, the Langmuir model is able to represent a wider class of diffusion phenomena than the Fick model. In particular, delay or, in contrary, fast absorption, at early instants of diffusion can be simulated with this model.

\subsection{Mechanical problem}

Concerning the deformation of the material submitted to local moisture content $c(\boldsymbol{x}, t)$ that occupies the domain $\Omega$ under small perturbations theory, plane strain assumption is retained. We denote by $\boldsymbol{u}(\boldsymbol{x}, t)$ the displacement field, by $\boldsymbol{\varepsilon}(\boldsymbol{u}(\boldsymbol{x}, t))$ the strain tensor, and by $\boldsymbol{\sigma}(\boldsymbol{x}, t)$ the Cauchy stress tensor. Both the reinforcements and the matrix are assumed to be linear isotropic elastic materials represented by the fourth order stiffness tensor $\mathbb{C}$ verifying 


$$
\mathbb{C}(\boldsymbol{x})= \begin{cases}\mathbb{C}_{m} & \text { if } \boldsymbol{x} \in \Omega_{m}, \\ \mathbb{C}_{r} & \text { if } \boldsymbol{x} \in \Omega_{r},\end{cases}
$$

where $\mathbb{C}_{m}$ and $\mathbb{C}_{r}$ are constant tensors. Moreover, $\beta_{h}$ is the hygroscopic expansion coefficient which is considered, in this work, identical in each direction. The hygroscopic expansion is represented by the diagonal tensor $\boldsymbol{\beta}_{h}$ whose diagonal components are equal to $\beta_{h}$. $\beta_{h}$ is taken equal to 0 for the hydrophobic reinforcements. Finally, the quasi-static linear elastic problem writes: find the displacement field $\boldsymbol{u}(\boldsymbol{x}, t)$ such that

$$
\begin{aligned}
& \nabla \cdot \boldsymbol{\sigma}=\mathbf{0} \text { on } \Omega, \\
& \boldsymbol{\sigma}=\mathbb{C}:\left[\boldsymbol{\varepsilon}-\boldsymbol{\beta}_{h} c\right] \text { on } \Omega, \\
& \boldsymbol{u}=\boldsymbol{u}_{i m p} \text { on } \Gamma_{u},
\end{aligned}
$$

where $\boldsymbol{u}_{i m p}$ is the imposed displacement on the part $\Gamma_{u}$ of $\partial \boldsymbol{\Omega}$ and $c(\boldsymbol{x}, t)$ is the solution field of either problem (4) or problem (6).

\section{Numerical aspects}

\subsection{Space and time discretizations and resolution}

Diffusion problems (4) and (6) and linear elastic problem (8) are solved using the classical Finite Element Method (FEM). At the spatial level, for each model the discretization is done using a finite element mesh composed of, about 240,000 3-nodes elements associated with linear interpolation functions. At the time level, the backward Euler integration scheme is used and the increment of time at each step is computed by the Abaqus ${ }^{\mathrm{TM}}$ solver. All computations in this work were performed in less than $4 \mathrm{~h}$ on a standard laptop computer. In practice, User ELement (UEL) dedicated to problems (4) and (6) were implemented in the industrial FEM code Abaqus $^{\mathrm{TM}}[30]$ allowing the use of its spatial and time solvers. Verification and convergence analyses, based on analytic solutions found in [24,26], were carried out to ensure the validity of the implementation.

\subsection{Generator of random geometries}

Industrial applications requires stiff materials, thus leads to consider a high volume fraction $v_{r}$ of the non penetrating reinforcements. Besides, a regular distribution of the fibers seems unlikely [27-29] and a random generator is then needed to create realistic geometries of the microstructure. Generators based on a rejection process of the fibers are hard to use since the targeted volume fraction is high [32]. Therefore, a specific procedure is used to circumvent this issue. The starting point is a regular (i.e. periodic) disposition of the fibers within the microstructure. The radius of the cylindrical fibers is deterministic and only their positions are disturbed with a procedure based on elastic shocks where the radial and tangential velocities, associated with each fiber, are independent random variables. Such a technique allows obtaining the desired random geometries with small computational times.

\section{Studies definition}

\subsection{Material properties}

The first matrix studied in this work is a Ciba Geigy LY 556 polyepoxy system, hardened by a HY 917 anhydride hardener and a DY 30 accelerator [33]. In the following paper, this resin will be referred as Epoxy A. The second one, which is also an epoxy based resin, hardened by an aliphatic polyamine [34], will be called Epoxy B. The diffusion parameters, presented in Table 1 for both Fick and Langmuir models, come from a minimization problem aiming at finding the optimal diffusion parameters. The
Table 1

Calculated diffusion parameters, for Fick and Langmuir models, and mechanical properties for Epoxy $A$ and Epoxy $B$.

\begin{tabular}{llllll}
\hline & \multicolumn{2}{l}{ Epoxy $A$} & & & Epoxy $B$ \\
\cline { 2 - 3 } \cline { 5 - 6 } & $\mathrm{F}$ & $\mathrm{L}$ & & $\mathrm{F}$ & $\mathrm{L}$ \\
\hline$D\left(\mu \mathrm{m}^{2} \mathrm{~s}^{-1}\right)$ & 0.082 & 2.04 & & 1.47 & 4.14 \\
$C^{\infty}(\%)$ & 1.71 & 1.71 & & 2.28 & 2.28 \\
$\alpha\left(10^{-6} \mathrm{~s}^{-1}\right)$ & $\mathrm{X}$ & 0.0717 & & $\mathrm{X}$ & 87.6 \\
$\beta\left(10^{-6} \mathrm{~s}^{-1}\right)$ & $\mathrm{X}$ & 0.0745 & & $\mathrm{x}$ & 53.4 \\
$\rho\left(\mathrm{Kg} / \mathrm{m}^{3}\right)$ & $1310[27]$ & & & & $1220[36,38]$ \\
$E(\mathrm{MPa})$ & $4000[36,38]$ & & & $2700[35]$ & \\
$v$ & $0.36[27]$ & & & $0.35[35]$ & \\
$\beta_{x}^{h}=\beta_{y}^{h}$ & $0.00324[27]$ & & & $0.001[35]$ & \\
\hline
\end{tabular}

least-square discrepancy between the experimental data, available in [33,34], and the analytic solutions, given in [24] for the Fick model and in [26] for the Langmuir model, is minimized. In order to use those solutions, the diffusion within the matrix, considered as an homogeneous material at this scale, is assumed unidirectional. Fig. 2 presents the sorption curve for the Epoxy A resin with the results for the Fick and the Langmuir models. It is obvious that Epoxy A presents an anomalous diffusion behavior. The agreement between simulations and experimental data is not good for the Fick model but is convincing for the Langmuir one. For these reasons, in the laminates $A$ analysis, only the Langmuir model will be considered. Figs. 3(a) and (b) illustrate the absorption curves obtained with the identified parameters of each diffusion model for Epoxy B. It can be seen on Fig. 3(a) that the two models give very close results. Differences can however be observed on Fig. 3(b) at the very first instants of the diffusion process. According to the experimental data, the Fick model overestimates the water content unlike the Langmuir model which better reproduces the diffusion in the beginning of the process (small delay). The Epoxy A resin is associated with hydrophobic reinforcements made of carbon while Epoxy $B$ is associated with glass fibers. The laminates based on Epoxy $A$ are named Laminates $A$ and, in the same manner, the laminates based on Epoxy $B$ are called Laminates $B$. Table 1 presents matrix mechanical properties while Table 2 presents the mechanical properties for the reinforcements. $E$ is the Young modulus and $v$ is the Poisson's ratio.

\subsection{Geometries of microstructure}

The computations realized in this work, are representative of typical water absorption specimens. The samples are square and

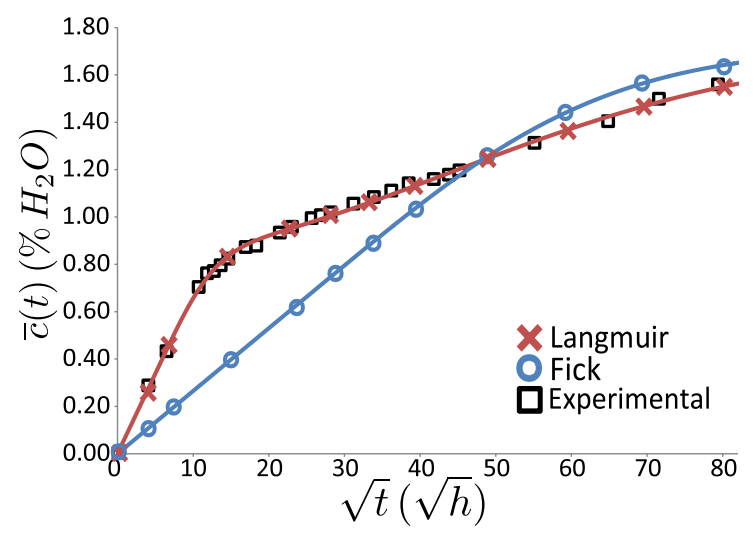

Fig. 2. Average water content $\bar{c}$ according to $\sqrt{t}$ in a pure resin sample of Epoxy A: experimental data (squares), Fick identification (circles) and Langmuir identification (crosses). 


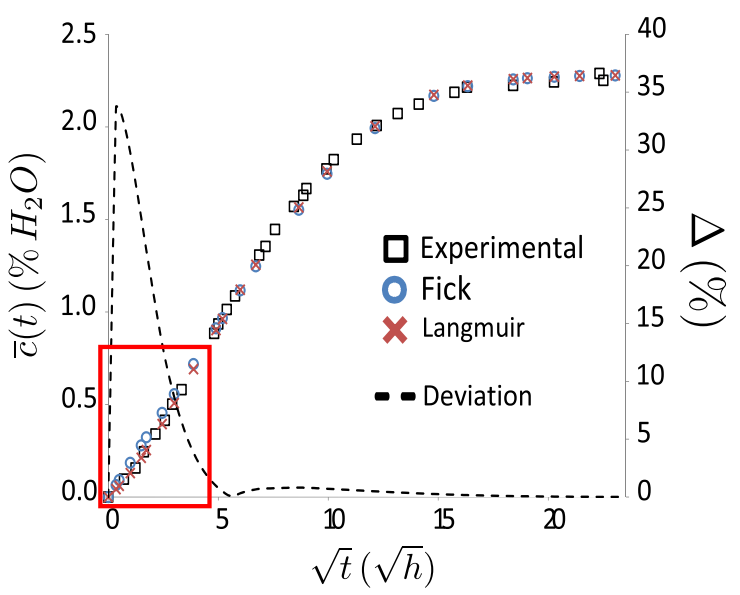

(a)

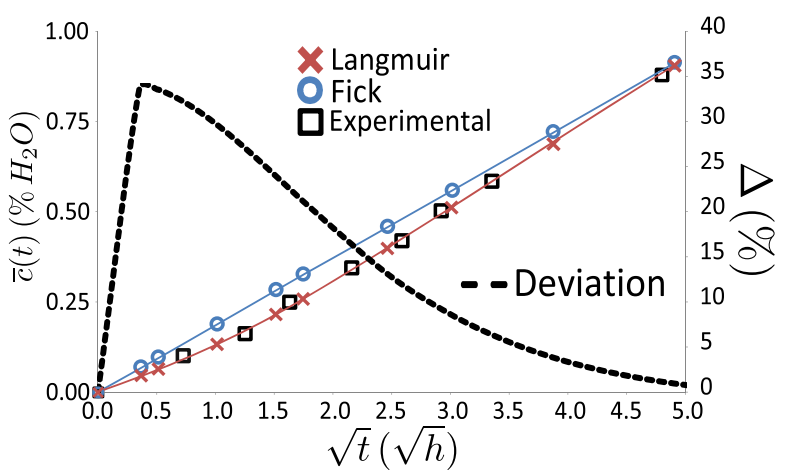

(b)

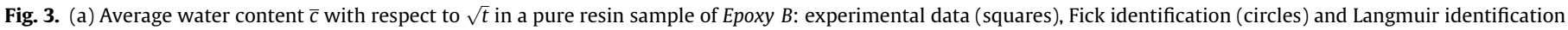
(crosses). $\Delta$ (dotted line) is the relative deviation between Fick and Langmuir models. (b) Focus on the first instants.

Table 2

Mechanical properties for carbon and glass reinforcements.

\begin{tabular}{lll}
\hline & Carbon & Glass \\
\hline$\rho\left(\mathrm{Kg} / \mathrm{m}^{3}\right)$ & $1780[27]$ & $2540[25,36,38]$ \\
$E(\mathrm{MPa})$ & $20000[27]$ & $72500[25,36,38]$ \\
$v$ & $0.33[27]$ & $0.22[36,38]$ \\
$\beta_{x}^{h}=\beta_{y}^{h}$ & 0 & 0 \\
\hline
\end{tabular}

the side is equal to $75 \mathrm{~mm}$. The thickness $e$, different in Laminates $A$ and Laminates $B$ analyses, is sufficiently small to neglect the water absorption by the edges. This assumption leads to a symmetrical diffusion problem. Therefore for computational resources considerations, only a slice of the superior half of the sample is numerically represented. We denote by $h$ the height and by $w$ the width of the numerical models. Geometries of microstructures are also described by the diameter of the fibers $d_{r}$ assumed identical and perfectly cylindrical, and by the volume fraction of reinforcements $v_{r}$. High volume fraction of reinforcements are retained in order to be representative of industrial applications. All parameters are presented in Table 3. For the first analysis, five different geometries are considered: one periodic and four others obtained with the random generator described in Section 3.2. Concerning the second analysis, two geometries are reviewed, a periodic and a random. Fig. 4(a) and (b) shows the geometries of microstructures used in the numerical simulations.

\subsection{Initial and boundary conditions}

Samples are initially considered dry, involving a zero moisture content within the matrix. The imposed moisture content $c_{i m p}$ applied on $\Gamma_{c}$ is taken equal to the maximum moisture content $C^{\infty}$ (cf. Table 1$)$. Symmetry conditions are used for the mechanical problem leading to dissociate $\Gamma_{u}$ into $\Gamma_{u_{X}}$ (symmetry along $X$-axis) and $\Gamma_{u_{Y}}$ (symmetry along $Y$-axis).

Table 3

Dimensional properties of the geometries of microstructures.

\begin{tabular}{lll}
\hline & Laminates $A$ & Laminates $B$ \\
\hline$w(\mu \mathrm{m})$ & 100 & 210 \\
$h(\mu \mathrm{m})$ & 600 & 1000 \\
$v_{r}(\%)$ & 61.0 & 53.3 \\
$d_{r}(\mu \mathrm{m})$ & 7 & 18 \\
\hline
\end{tabular}

\section{Results and discussion}

\subsection{Laminates $A$}

\subsubsection{Local behavior}

Fig. 5(a) and (b) shows the local stress fields $\sigma_{y y}$, in the righthand corner of each geometry, after $1 \mathrm{~h}$ and when the steady state is reached. It is worth noting that the extreme stresses are localized in the matrix. The materials points surrounded by fibers are in traction while the other points placed in a zone with a lack of reinforcements are under compression. Moreover, those two figures highlight the geometrical impact on stress intensity. The maximum stress is reached at steady state where the hygroscopic strain is maximal. If the range of stress is similar in all Random cases, compared to the ones from the Periodic geometry they are about 3 times higher. In addition to emphasize the geometrical impact, Fig. 6 presents the density distribution of the stress component in the matrix $\sigma_{y y}^{m}$ for each geometry. It highlights that Random instances give close results and they have wide stress distribution, $-70 \mathrm{MPa}<\sigma_{y y}^{m}<110 \mathrm{MPa}$, while the Periodic one is tighter, $-40 \mathrm{MPa}<\sigma_{y y}^{m}<40 \mathrm{MPa}$. This is particularly important to emphasize that in this work, due to the use of an uncoupled model, the local moisture content fields are equal at steady state. Nevertheless, this will no longer be true when a coupling between mechanical and diffusion phenomena is added. Furthermore, the maximum stress reached in the matrix indicates that damage might occur without adding any external loads. This phenomenon is described in the literature $[17,33,35]$ and might be disastrous for the structure.

\subsubsection{Global behavior}

Fig. 7 presents the sorption curve for the Laminates A geometries. All geometries give closed sorption curve even for the Periodic microstructure, despite small deviation may be seen at the first instants where the diffusion is faster in the Periodic case. Indeed, as related in literature [29] fibers cluster acts as barrier. Moreover, it may be noticed that the sorption curves predicted for the composites structure are typical of an anomalous diffusion behavior and furthermore their shape is similar to that of the pure resin sample (Fig. 2). The fact that resin and composite samples behaved in the same manner is noticeable in the literature for polyester [16] and for epoxy system [33].

Fig. 8 presents the spatial mean stress into the matrix $\overline{\sigma_{y y}^{m}}$ and within the reinforcements $\overline{\sigma_{y y}^{r}}$. Firstly, it shows that the matrix is 


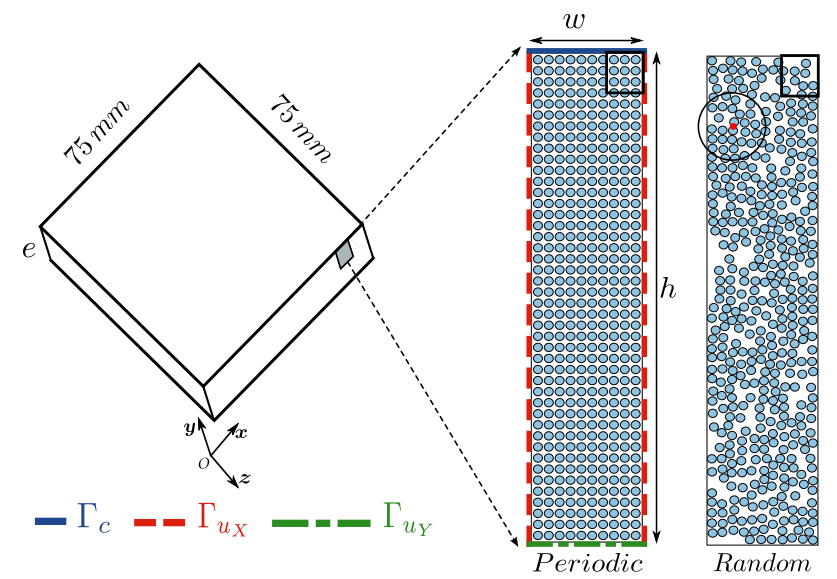

(a)

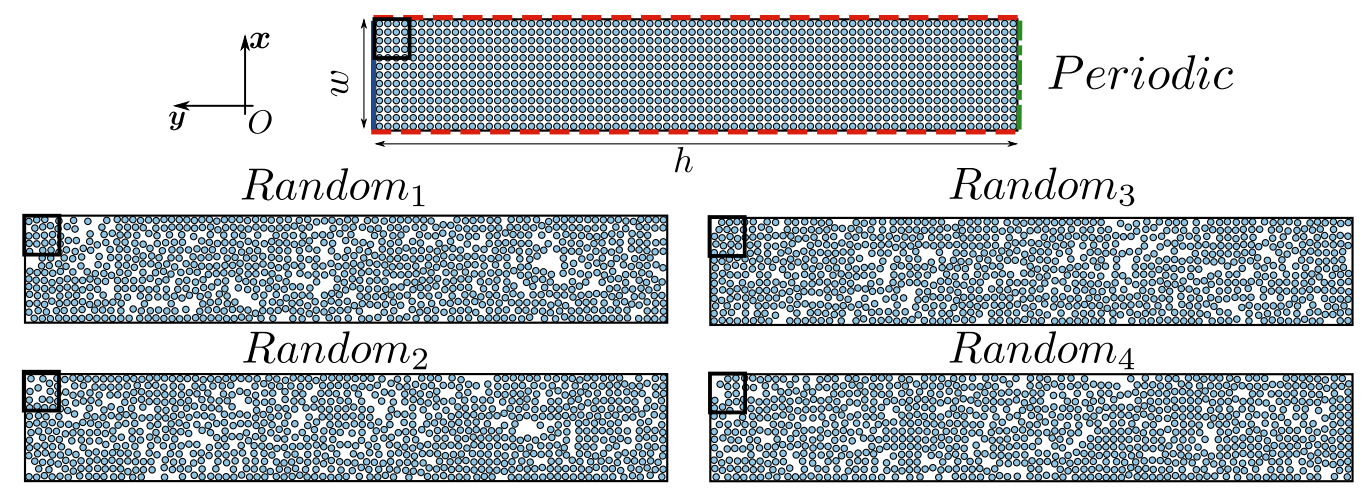

(b)

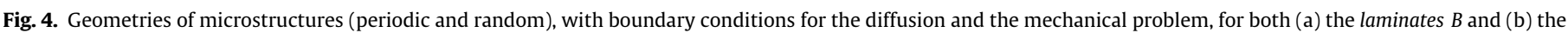
laminates $A$ analyses.
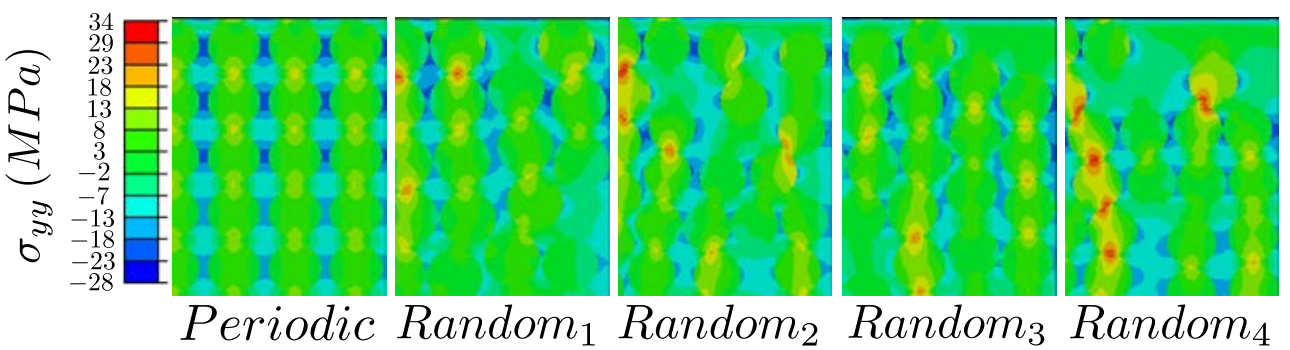

(a)
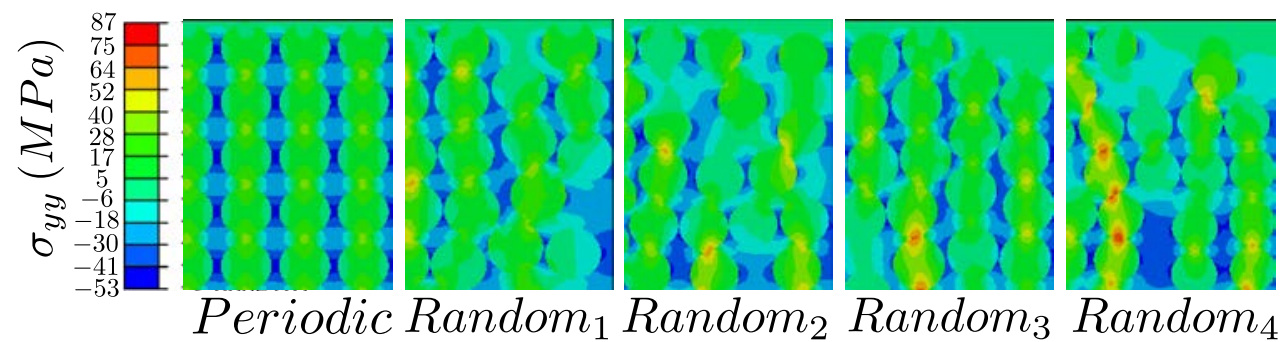

(b)

Fig. 5. Local stress fields $\sigma_{y y}$ (a) at $t=1 \mathrm{~h}$ and (b) when the steady state is reached.

globally under compression while the reinforcements are in tension. However, compared to observations made on Fig. 6, it highlights the loss of information resulting from considering the mean values. Indeed, the matrix could locally be in tension or in compression while the mean stress is negative. The converses is also true for the reinforcements. Secondly, it is worth noting that 


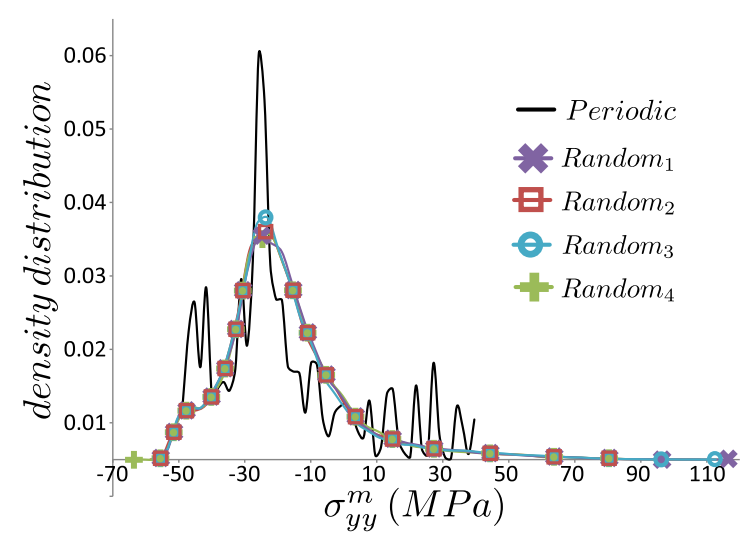

Fig. 6. Density distribution of the stress in the matrix $\sigma_{y y}^{m}$ for each geometry at steady state.

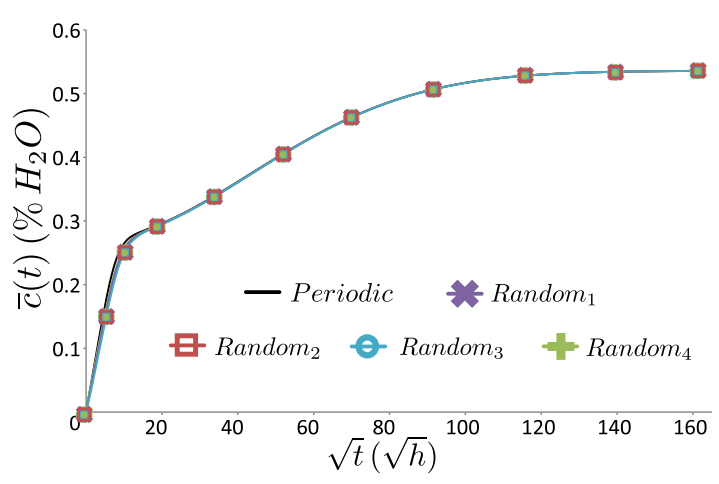

Fig. 7. Average water content $\bar{c}(t)$ according to $\sqrt{t}$ in the Laminates $A$.

mean stress intensity are very closed except at steady state where small discrepancies appear. In fact, a deviation of 0.5 MPa exists between the two extremes instances.

This first analysis points out the use of the improved Langmuir diffusion model in order to perform a numerical investigation of the humid aging of composite materials. In addition, the geometrical effect on local field, particularly for the stresses induced by the swelling of the matrix, between Periodic and Random geometries is highlighted.

\subsection{Laminates $B$}

\subsubsection{Local behavior}

We now focus on the second application Laminates $B$ where the resin can be well represented with both diffusion models, namely:

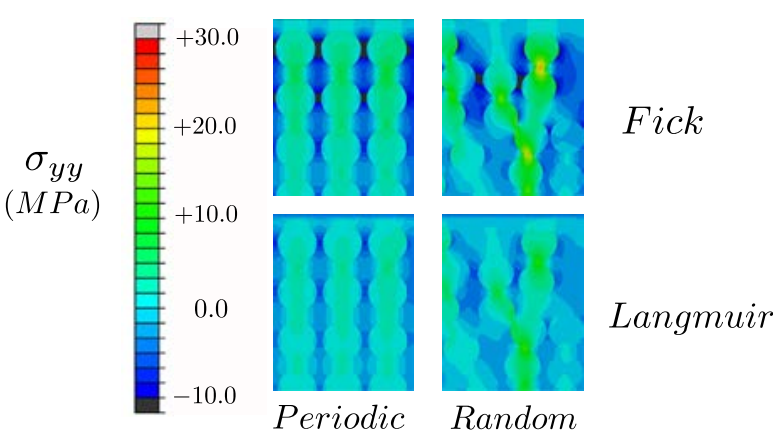

Fig. 9. Stress fields $\sigma_{y y}$ in the top right-hand corner of the two geometries obtained with Fick and Langmuir models at $t=1 \mathrm{~h}$.

either Fick or Langmuir. Fig. 9 illustrates the stress fields $\sigma_{y y}$ in the top right-hand corner of the two considered geometries at $t=1$ hour which is representative of the transient part of the diffusion process, where a deviation occurs in the sorption curves predicted by the two models for the pure resin sample. The Fick model leads to higher predicted local stress. Those differences are explained by the mismatch of the moisture content fields obtained with the two models. The local moisture content is indeed higher with the Fick model for two combined reasons. Firstly, all the water molecules participate to the diffusion process with this model. Secondly, the sorption curve identified with the Fick model leads to a faster diffusion in the matrix during the first instants as shown in Fig. 3(b). In addition to this observation, Fig. 10(a) and (b) shows the moisture absorption and the evolution of $\sigma_{y y}$ according to $\sqrt{t}$ at a specific material point. It emphasizes the local mismatch between the moisture content and its impact on local stresses. It may be noted that at this particular material point, the matrix is in tension due to the fact that it is confined between close fibers. It is worth noting that the shape of those curves is similar and the maximum deviation for the stress field, between the two models, is about $10 \mathrm{MPa}$, which is a high value compared to the ultimate tensile strength of this kind of material $\sigma_{u} \approx 55 \mathrm{MPa}$ $[25,33,36]$. Besides, as shown in the first analysis, we also notice a geometrical influence on the stress fields. For instance, the difference between the maximum local stress obtained with each model are enhanced when the fibers are randomly distributed. In fact, this maximum is $3 \mathrm{MPa}$ for the Periodic case whereas it is $18 \mathrm{MPa}$ for the Random case. These higher stresses are due to closer fibers. Fig. 11 presents the stress fields $\sigma_{y y}$ when the steady state is reached for each geometry. As in the first analysis, the geometry clearly impacts the local stress field for which significant discrepancies can be observed. In addition, since we only use an

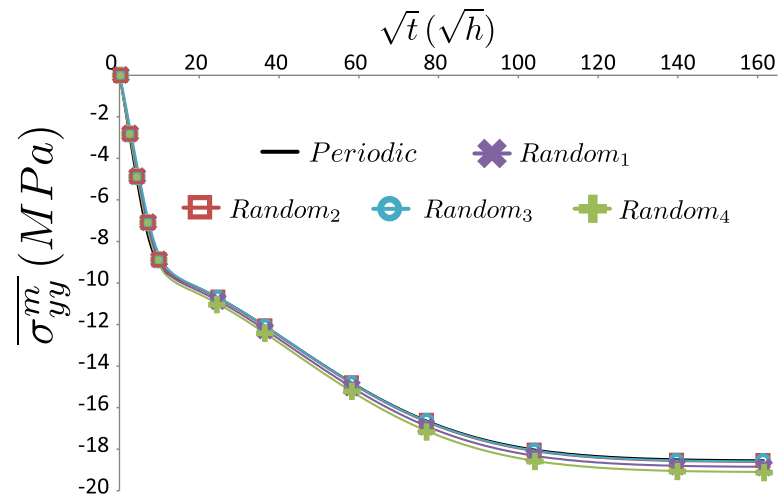

(a)

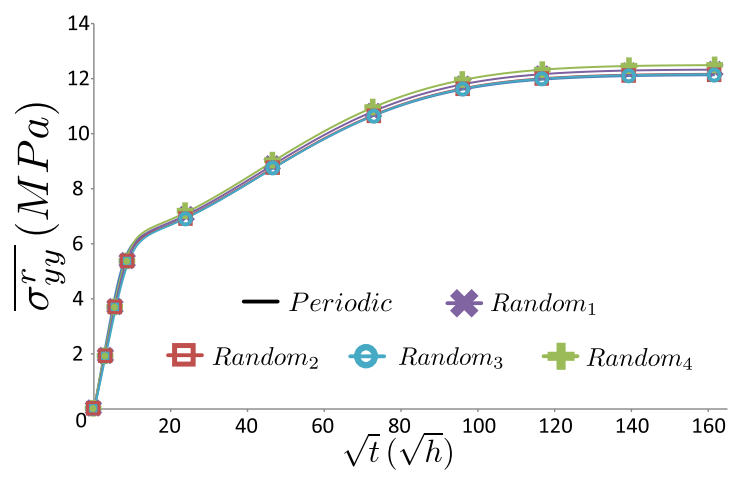

(b)

Fig. 8. Mean stress (a) inside the matrix $\overline{\sigma_{y y}^{m}}$ and (b) inside the reinforcements $\overline{\sigma_{y y}^{r}}$ with respect to $\sqrt{t}$ for the Laminates A. 


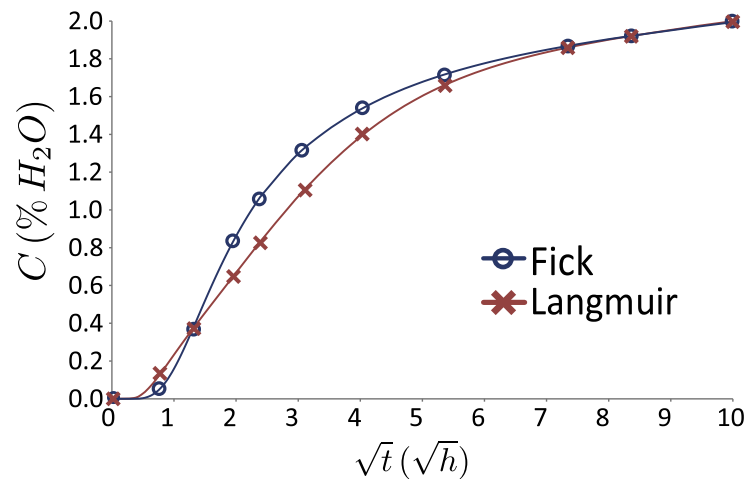

(a)

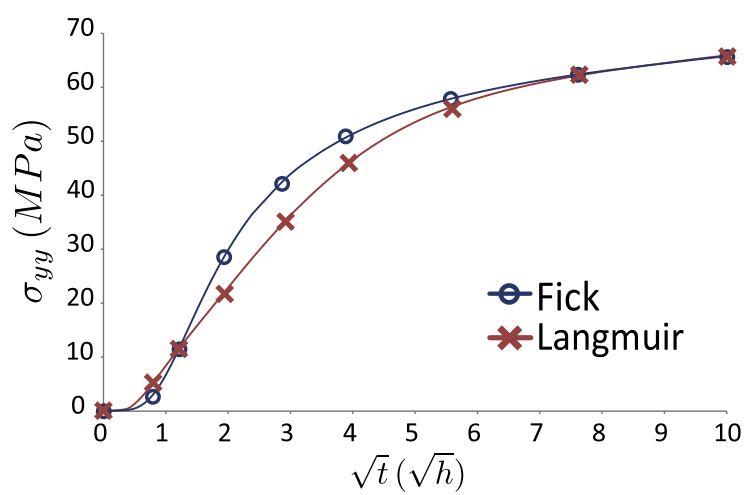

(b)

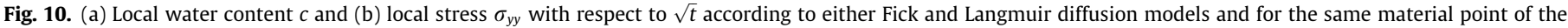
Random geometry.

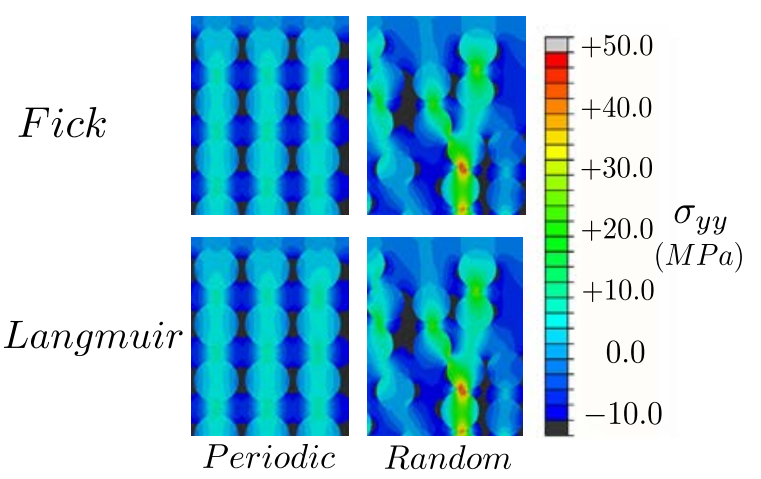

Fig. 11. Stress fields $\sigma_{y y}$ in the top right-hand corner of the two configurations and the two models at steady state.

uncoupled hygro-elastic model, both diffusion models lead to the same moisture contents and stationary stress fields. However, adding a coupling between the diffusion and the mechanical state $[25,37]$ and/or a local mechanical model, such as a damage model $[17,25]$, could affect this observation. Further works will be devoted to the investigation of such local couplings.

\subsubsection{Global behavior}

Henceforth, we focus on spatial average quantities. Fig. 12(a) shows the evolution of the average content $\bar{c}(t)$ obtained with both models and for the two geometries. We can observe that both models conduct to very close results. Only small differences as the ones observed for the matrix diffusion behavior appear at the first instants of the diffusion (Fig. 12(b)). Moreover, changes in the geometry have small effects on the evolution of the average moisture content. We then analyze the spatial average stress within the matrix and the reinforcements respectively denoted by $\overline{\sigma_{y y}^{m}}$ and $\overline{\sigma_{y y}^{r}}$. Fig. 13 presents the evolution of $\overline{\sigma_{y y}^{m}}$ and $\overline{\sigma_{y y}^{r}}$ according to $\sqrt{t}$ for each geometry. Firstly, as for $\bar{c}(t)$, the two models give close results except at the beginning of the diffusion process. Secondly, it is worth noting that the matrix phase is in a global compression state when the fibers are in a global traction state. Thirdly, it is important to highlight the deviation between the different geometries: this actually confirms the findings of the first analysis. In particular, the Periodic case presents a maximum relative deviation of $30 \%$ corresponding to a gap of $\approx 1.5 \mathrm{MPa}$. The higher deviation on global stress values occurring in this analysis may be explained by the lower volume fraction of reinforcements which leads to less stress in the Periodic instance. This indicates that a

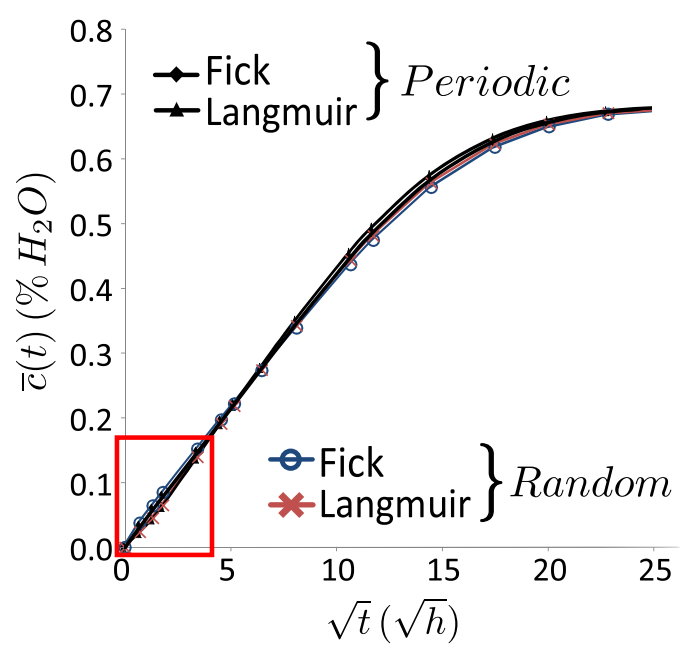

(a)

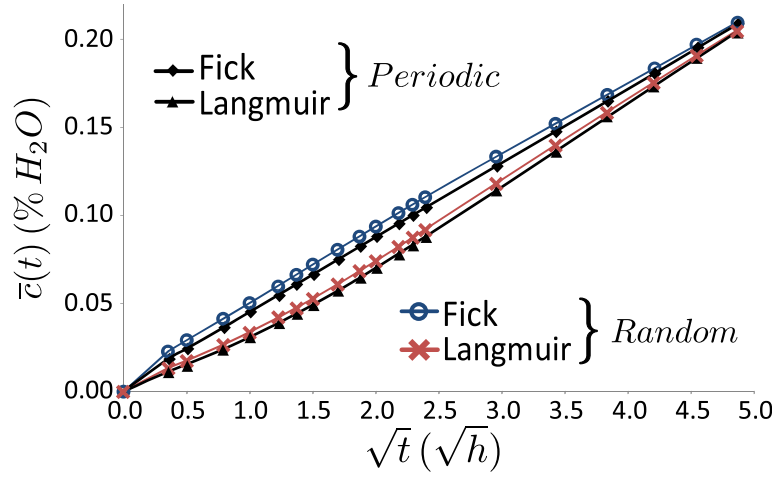

(b)

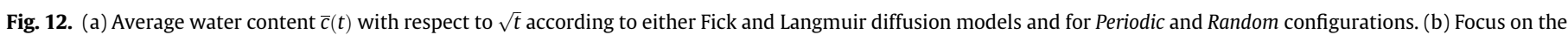
first instants. 


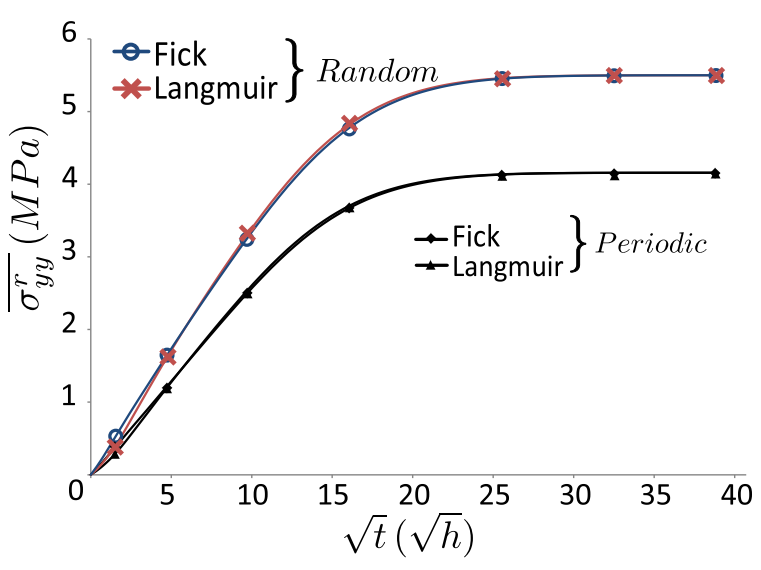

(a)

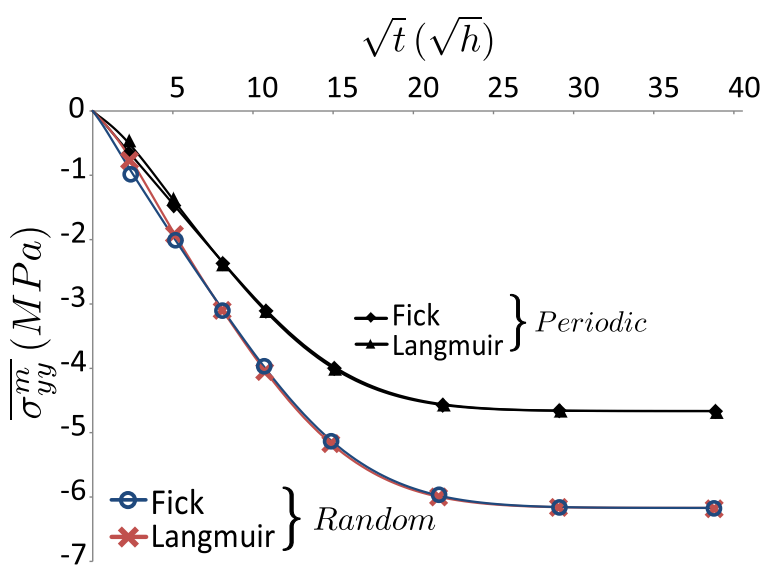

(b)

Fig. 13. Mean stress (a) inside the reinforcements $\overline{\sigma_{y y}^{r}}$ and (b) inside the matrix $\overline{\sigma_{y y}^{m}}$ with respect to $\sqrt{t}$ for the two geometries.

more precise analysis of the effect of the volume fraction of reinforcements and the distribution of the fibers is needed.

To sum up, the choice between the model of diffusion studied in this work, considering uncoupled behavior, can have a noticeable impact on local fields erased when spatial average quantities are considered. This indicates that local investigations at microscopic level are disturbed by the choice on the water diffusion model unlike studies about scale transition process.

\section{Conclusion}

In this work, we have proposed a transient uncoupled hygroelastic analysis of two polymer resins reinforced with hydrophobic glass and carbon fibers. Firstly, we analyze an epoxy system presenting a non-Fickian diffusion behavior and therefore, solely the Langmuir model was used. Using a dedicated random generator, effects of the geometrical distribution of the fibers have also been discussed. Secondly, we study a resin which has a diffusive behavior almost Fickian. Both the Fick and the Langmuir diffusion models, identified on experimental data of this polymer, have been compared through numerical simulations. Those analyses were possible through the implementation of the Langmuir diffusion model inside the commercial Finite Element Code Abaqus ${ }^{\mathrm{TM}}$. The geometrical effect on local stress field is emphasized in the first part. Concerning the second part, analyses on both local and global quantities have shown discrepancies between the two models during the transient part of the moisture diffusion process whereas only the geometry affects the steady state results. The higher stresses are mainly localized where the matrix is trapped in fibers clusters. Without achieving scale transition modeling, spatial average quantities have been studied. The loss of information using those procedure was emphasized and further works will be devoted to (i) analyze diffusion-mechanical couplings and (ii) use scale transition methods to take into account microscopic details at a higher scale.

\section{Acknowledgments}

The authors acknowledge the IRT Jules Verne (French Institute in Research and Technology in Advanced Manufacturing Technologies for Composite, Metallic and Hybrid Structures) and the industrial partners (ALSTOM, CETIM, DCNS and STX France) of the collaborative project ECOSAM2 (on the lightening of marine structure and super-structure), for their financial support.

\section{References}

[1] Zhou Z, Benbouzid M, Charpentier JF, Scuiller F, Tang T. A review of energy storage technologies for marine current energy systems. Renewable Sustainable Energy Rev 2013;18:390-400.

[2] Perveen R, Kishor N, Mohanty S. Off-shore wind farm development: present status and challenges. Renewable Sustainable Energy Rev 2014;29:780-92.

[3] Motley M, Barber R. Passive control of marine hydrokinetic turbine blades. Compos Struct 2014:110:133-9.

[4] Mouritz A, Geller E, Burchill P, Challis K. Review of advanced composite structures for naval ships and submarines. Compos Struct 2001;53:21-41.

[5] Grogan D, Leen S, Kennedy C, O Bradaigh C. Design of composite tidal turbine blades. Renewable Energy 2013;57:151-62.

[6] Grabovac I. Bonded composite solution to ship reinforcement. Compos Part A 2003;34:847-54.

[7] Nicholls-Lee R, Turnock S, Boyd S. Application of bend-twist coupled blades for horizontal axis tidal turbines. Renewable Energy 2013;50:541-50.

[8] Bosia F, Botsis J, Facchini M, Giaccari P. Deformation characteristics of composite laminates. Part I: Speckle interferometry and embedded Bragg grating sensor measurements. Compos Sci Technol 2002;62:41-54.

[9] Parlevliet PP, Bersee HE, Beukers A. Measurement of (post-)curing strain development with fibre Bragg gratings. Polym Test 2010;29:291-301.

[10] Yagoubi JE, Lubineau G, Roger F, Verdu J. A fully coupled diffusion-reaction scheme for moisture sorption-desorption in an anhydride-cured epoxy resin. Polymer 2012;53:5582-95.

[11] Vaddadi P, Nakamura T, Singh RP. Transient hygrothermal stresses in fiber reinforced composites: a heterogeneous characterization approach. Acta Mater 2003;51:177-93.

[12] Chateauminois A, Vincent L, Chabert B, Souli J. Study of the interfacial degradation of a glass-epoxy composite during hygrothermal ageing using water diffusion measurements and dynamic mechanical thermal analysis. Polymer 1994;35:4766-74.

[13] Olmos D, Lopez-Moron R, Gonzalez-Benito J. The nature of the glass fibre surface and its effect in the water absorption of glass fibre/epoxy composites, the use of fluorescence to obtain information at the interface. Compos Sci Technol 2006;66:2758-68.

[14] Selzer R, Friedrich K. Mechanical properties and failure behaviour of carbon fibre-reinforced polymer composites under the influence of moisture. Compos Part A 1997;28:595-604.

[15] Sala G. Composite degradation due to fluid adsorption. Compos Part B 2000;31:357-73.

[16] Visco A, Calabrese L, Cianciafara P. Modification of polyester resin based composites induced by seawater absorption. Compos Part A 2012;36:38-43.

[17] Abhilash A, Joshi SP, Mukherjee A, Mishnaevsky Jr L. Michromechanics of diffusion-induced damage evolution in reinforced polymers. CompSciTech 2010;71:333-42.

[18] Gladwell G. Durability of composites in a marine environment. 1st ed. Springer; 2014.

[19] Shen C, Springer G. Moisture absorption and desorption of composite materials. J Compos Mater 1975;10:2-20.

[20] Loos AC, Springer GS. Moisture absorption of graphite-epoxy composites immersed in liquids and in humid air. J Compos Mater 1979;13:131-47.

[21] Loos AC, Springer GS. Moisture absorption of polyester-e glass composites. J Compos Mater 1979;14:142-54.

[22] Roy S, Xu W, Park S, Liechti K. Anomalous moisture diffusion in viscoelastic polymers: modeling and testing. J Appl Mech 2000;67:391-6.

[23] Popineau S, Rondeau-Mouro C, Sulpice-Gaillet C, Shanahan M. Free/bound water absorption in an epoxy adhesive. Polymer 2005;46:10733-40. 
[24] Crank J. The mathematics of diffusion. 2nd ed. Oxford University Press; 1975.

[25] Weitsman YJ. Fluid effects in polymers and polymeric composites. 1st ed. Springer; 2012.

[26] Carter H, Kibler K. Langmuir-type model for anomalous moisture diffusion in composite resins. J Compos Mater 1978:12:118-31.

[27] Vaddadi P, Nakamura T, Singh RP. Transient hygrothermal stresses in fibe reinforced composites: a heterogeneous characterization approach. Compos Part A 2003;34:719-30.

[28] Bond DA. Moisture diffusion in a fiber-reinforced composite. Part I: NonFickian transport and the effect of fiber spatial distribution. J Compos Mater 2005;39:2113-29.

[29] Joliff Y, Belec L, Chailan J. Modified water diffusion kinetics in an unidirectiona glass/fibre composite due to the interphase area: experimental, analytical and numerical approach. Compos Struct 2013;97:296-303.

[30] D.S.S. Corp, ABAQUS V6.12: Abaqus User Subroutines Reference Manual; 2012

[31] Ramezani Dana H, Perronnet A, Fréour S, Casari P, Jacquemin F. Identification of moisture diffusion parameters in organic matrix composites. J Compos Mater 2013;47:1081-92.
[32] Torquato S. Random heterogeneous materials: microstructure and macroscopic properties. Springer; 2002.

[33] Perreux D, Suri C. A study of the coupling between the phenomena of water absorption and damage in glass/epoxy composite pipes. Compos Sci Technol 1997:57:1403-13.

[34] Joliff Y, Belec L, Heman M, Chailan J. Experimental, analytical and numerical study of water diffusion in unidirectional composite materials - interphase impact. Comput Mater Sci 2012;64:141-5.

[35] Joliff Y, Rekik W, Belec L, Chailan JF. Study of the moisture/stress effects on glass fibre/epoxy composite and the impact of the interphase area. Compos Struct 2014;108:876-85.

[36] Herakovich CT. Mechanics of fibrous composites. Wiley; 1998.

[37] Sar B-E, Fréour S, Davies P, Jacquemin F. Coupling moisture diffusion and internal mechanical states in polymers: a thermodynamical approach. Eur J Mech A/Solids 2013;50:541-50.

[38] Gay D, Hoa SV, Tsai SW. Composite materials: design and applications. 4th ed. CRC Press; 2003. 